\title{
Neutral Black Rings in Five Dimensions are Unstable
}

\author{
Jorge E. Santos ${ }^{*}$ and Benson Way ${ }^{\dagger}$ \\ DAMTP, Centre for Mathematical Sciences, University of Cambridge, Wilberforce Road, Cambridge CB3 OWA, United Kingdom
}

(Received 9 March 2015; published 3 June 2015)

\begin{abstract}
We study nonaxisymmetric linearized gravitational perturbations of the Emparan-Reall black ring using numerical methods. We find an unstable mode whose onset lies within the "fat" branch of the black ring and continues into the "thin" branch. Together with previous results using Penrose inequalities that fat black rings are unstable, this provides numerical evidence that the entire black ring family is unstable.
\end{abstract}

DOI: 10.1103/PhysRevLett.114.221101

PACS numbers: 04.50.Gh, 04.25.dc, 04.70.-s

During the golden age of general relativity, fourdimensional black holes were shown to be remarkably featureless. The Kerr black hole is the most general asymptotically flat black hole solution to the vacuum Einstein equation [1]. It has spherical topology and depends only on two parameters: the mass $M$ and the angular momentum $J$ [2,3]. Moreover, for specific values of $M$ and $J$, there is only one such black hole; i.e., the Kerr black hole is unique. Additionally, its linear stability has been shown for all modes [4]. Numerical simulations suggest that Kerr black holes are also nonlinearly stable, though a complete proof remains elusive (see, however, [5-7]). These facts have led to the conjecture that "four-dimensional black holes have no hair".

There is a natural higher-dimensional extension of the Kerr black hole-the Myers-Perry black hole [8]. Like the Kerr black hole, it has spherical topology, depends uniquely on the mass and angular momenta, and (for sufficiently slow rotation) has substantial evidence for its stability $[9,10]$.

Nevertheless, after the initial discovery of the black ring by Emparan and Reall $[11,12]$, it became clear that black holes in higher dimensions are dramatically different from those in four dimensions. These black rings are fivedimensional solutions with topology $S^{1} \times S^{2}$ and come in two types described by their shape: "fat" rings and "thin" rings. The two branches of solutions are distinct except for a unique ring that is both fat and thin. For every fat ring, there is a thin ring and a Myers-Perry black hole with the same mass and angular momentum. Thus, uniqueness is broken among black rings but also with Myers-Perry black holes. In light of this, a higher-dimensional no-hair theorem seems unlikely to hold.

Yet, another surprise in higher dimensions is the existence of a new type of instability. The so-called GregoryLaflamme instability was first found in black strings [13], but there are analogous instabilities for rapidly rotating Myers-Perry black holes [10,14-17]. The general picture is that black objects with extended directions are unstable to perturbations along those extended directions.

Since many of the solutions that violate uniqueness have extended directions, there is hope that the spirit of the no-hair theorems can be restored. That is, there is a unique stable solution which is a slowly rotating Myers-Perry black hole-a dynamical no-hair conjecture.
In five dimensions, the (in)stability of the black ring is a natural setting to test this conjecture. Indeed, black rings violate uniqueness, but moreover all known solutions in five dimensions are either Myers-Perry black holes or contain a topologically $S^{1} \times S^{2}$ black object as a horizon component.

Fat rings were conjectured to be unstable in Refs. $[18,19]$ and confirmed in Ref. [20] by using local Penrose inequalities. However, little is known about the stability of thin rings, except that very thin rings ought to be unstable to the Gregory-Laflamme instability, because they resemble boosted black strings [11-13,21]. Though this argument is physically sound, it says little about the stability of thin, but not very thin, rings (which happens to coincide with where black rings violate uniqueness with themselves and Myers-Perry black holes.) Our goal is to demonstrate that such a window of stability does not exist by computing gravitational perturbations of the black ring.

Numerical approach.-We begin by presenting coordinates for the black ring that we found suitable for numerics:

$$
\begin{aligned}
d s^{2}= & R^{2}\left\{-\left(1-y^{2}\right)^{2} F d t^{2}\right. \\
& +\frac{k_{0}^{2}}{h^{4}}\left[\frac{4}{2-y^{2}} \frac{f_{2}}{g} d y^{2}+y^{2}\left(2-y^{2}\right) S\left(d \psi-\Omega h^{4} W d t\right)^{2}\right. \\
& \left.\left.+\frac{4 \beta^{2}}{2-x^{2}} \frac{f_{2}}{f_{1}} d x^{2}+\beta^{2} x^{2}\left(2-x^{2}\right)\left(1-x^{2}\right)^{2} f_{1} d \phi^{2}\right]\right\}
\end{aligned}
$$

where the functions $f_{1}, f_{2}, g, h, F, S$, and $W$ are, respectively,

$$
\begin{aligned}
f_{1} & =1+\beta^{2} x^{2}\left(2-x^{2}\right), \quad f_{2}=1+\alpha^{2} \beta^{2} x^{2}\left(2-x^{2}\right) \\
g & =1+\frac{y^{2}\left(2-y^{2}\right)}{\beta^{2}}, \quad h=\sqrt{\beta^{2} x^{2}\left(2-x^{2}\right)+y^{2}\left(2-y^{2}\right)} \\
F & =\frac{f_{2} g}{\left[\alpha^{2} f_{1}^{2}-\left(1-y^{2}\right)^{2}\left(\alpha^{2}-1\right)\right] g+\alpha^{2}\left(\alpha^{2}-1\right) h^{4}} \\
S & =\frac{g}{F}, \quad W=\frac{\alpha^{2}\left(1+\alpha^{2} \beta^{2}\right)}{\beta^{2} f_{2} g} F
\end{aligned}
$$

the constants $\Omega$ and $k_{0}$ are, respectively, 


$$
\Omega=\frac{\beta^{2} \sqrt{\alpha^{2}-1}}{1+\beta^{2}}, \quad k_{0}=\frac{1+\beta^{2}}{\alpha \beta \sqrt{1+\alpha^{2} \beta^{2}}},
$$

and $\alpha \geq 1, \beta>0$ are constants that parametrize this family of solutions (the constant $R$ merely sets a scale). These parameters are related to the more familiar ones in Ref. [12] via

$$
\nu=\frac{\beta^{2}}{2+\beta^{2}}, \quad \lambda=\frac{\alpha^{2} \beta^{2}}{2+\alpha^{2} \beta^{2}},
$$

which satisfy $0<\nu \leq \lambda<1$. The parameters $\lambda$ and $\nu$ are more prevalent in the literature, so we will refer to these rather than $\alpha$ and $\beta$. The remainder of the line element in Ref. [12] can be reproduced by the redefinitions

$$
\begin{aligned}
& \tilde{x}=1-2\left(1-x^{2}\right)^{2}, \quad \tilde{y}=-\frac{1-(1-\nu)\left(1-y^{2}\right)^{2}}{\nu}, \\
& \tilde{t}=R t, \quad \tilde{\psi}=\frac{\sqrt{1-\lambda}}{1-\nu} \psi, \quad \tilde{\phi}=\frac{\sqrt{1-\lambda}}{1-\nu} \phi, \\
& \tilde{R}=\frac{\left(1+\beta^{2}\right) \sqrt{2+\alpha^{2} \beta^{2}}}{\alpha \beta^{2} \sqrt{2+\beta^{2}} \sqrt{1+\alpha^{2} \beta^{2}}} R,
\end{aligned}
$$

where quantities with a tilde refer to those in Ref. [12].

Our coordinates range in $x \in[0,1]$ and $y \in[0,1]$ with the axis of rotation at $y=0$, the horizon at $y=1$, the outer axis of the ring at $x=0$, and the inner axis at $x=1$. Asymptotic infinity is at $x=y=0$, which corresponds to $h=0$. The period of $\psi$ and $\phi$ are set to $2 \pi$, and the temperature of the horizon is $1 /(2 \pi)$.

Unless $\lambda=2 \nu /\left(1+\nu^{2}\right)$, there will be a conical singularity at the inner axis. We shall see that the singular solutions will be useful to us. In particular, there is a static solution when $\lambda=\nu$. We are chiefly interested in the nonsingular family of solutions which we call the "balanced" ring. When the ring is balanced, $0<\nu \leq 1 / 2$ are the thin rings, while $1 / 2 \leq \nu<1$ are the fat rings.

We wish to study gravitational perturbations of (1). We write the perturbed metric as $g_{a b}={ }^{0} g_{a b}+h_{a b}$, where ${ }^{0} g_{a b}$ refers to the background solution (1) and $h_{a b}$ is our metric perturbation. Since we are working with the vacuum Einstein equation, we are free to impose the transversetraceless gauge condition

$$
\nabla^{a} h_{a b}=0, \quad h^{a}{ }_{a}=0,
$$

where, as elsewhere in this Letter, covariant differentiation and the raising and lowering of indices are done with respect to the background ${ }^{0} g_{a b}$. In this gauge, the linearized Einstein equation is

$$
\left(\Delta_{L} h\right)_{a b} \equiv-\nabla_{c} \nabla^{c} h_{a b}-2 R_{a}^{c}{ }^{c}{ }^{d} h_{c d}=0,
$$

where $\Delta_{L}$ is the Lichnerowicz operator.
Thin rings are extended in the $\partial_{\psi}$ direction, so the Gregory-Laflamme instability would break the $\partial_{\psi}$ symmetry. For simplicity, we preserve the remaining symmetry $\partial_{\phi}$. We therefore perform a mode decomposition $h_{a b}=e^{-i \omega t+i m \psi} \tilde{h}_{a b}$, where $\tilde{h}_{a b}$ are functions of $x$ and $y$.

Our background has a fixed temperature $T=1 /(2 \pi)$, so the frequency $\omega$ is equivalent to the more general dimensionless quantity $\varpi \equiv \omega /(2 \pi T)$. Incidentally, this quantity is equivalent to $\varpi=\left(R_{\text {out }}-R_{\text {in }}\right) \omega$, where $R_{\text {out }}$ and $R_{\text {in }}$ are the outer and inner $S^{1}$ equatorial horizon radii, respectively.

Since the $m=0$ modes do not break the rotation axis and $m=1$ modes were not found to be unstable in other systems with nonaxisymmetric instabilities [10], we will for definitiveness and simplicity set $m=2$.

Preserving $\partial_{\phi}$ symmetry lets us set $h_{\mu \phi}=0$ for $\mu \neq \phi$. This leaves 11 functions, one of which can be removed by imposing tracelessness. After imposing tracelessness, there are six components of (7) which together with the four nontrivial components of the transverse condition in (6) form a set of ten independent equations. The remaining components of (7) can be derived from this set.

Our task is to solve this set of ten, two-dimensional partial differential equations in the form of a quadratic eigenvalue problem in $\omega$. As boundary conditions, we impose regularity on the outer axis and axis of rotation, ingoing boundary conditions at the horizon, and outgoing boundary conditions at infinity. On the inner axis, we demand that the conical excess or deficit does not change, which is equivalent to regularity when there is no conical singularity.

This problem is complicated by the fact that infinity is at the coordinate singularity $x=y=0$. Because of this singularity, approaching the point $x=y=0$ from different directions will yield different values for $h_{a b}$. To obtain a well-posed problem, our remedy is to use a different coordinate system near infinity given by

$$
\rho=h, \xi=\sqrt{1-\frac{\beta x \sqrt{2-x^{2}}}{h}},
$$

where the function $h$ was given in (2). In these coordinates, spatial infinity is at the hyperslice $\rho=0$. We divide our domain into two nonoverlapping coordinate patches: one in $(\rho, \xi)$ coordinates containing infinity and another in $(x, y)$ coordinates containing the horizon and the inner axis. Grids can then be placed on these patches by using transfinite interpolation. We must also impose additional patching conditions that require that $h_{a b}$ and its first derivatives match on patch boundaries.

Once the problem is discretized by such a patched grid (we use pseudospectral collocation on Chebsyshev grids), it is reduced to a quadratic eigenvalue problem in linear algebra, which can be solved on a computer. Unfortunately, because of the size of the matrices required, a direct computation of the spectrum (reduction to a linear matrix 
pencil followed by $Q Z$ factorization) yields a large number of nonphysical spurious modes. We were unable to extract the physical modes from such a spectrum.

We must therefore seek an alternate route. Rather than the entire spectrum, we wish to obtain only the unstable Gregory-Laflamme mode of the balanced ring. By varying the parameters of the black ring, it is natural to suspect that this mode is connected to some Gregory-Laflamme mode of the static ring, the onset of which is a zero frequency mode $(\omega=0)$.

Since we suspect that our desired mode is connected to a zero frequency mode, consider the more general problem (with the same gauge and boundary conditions)

$$
\Delta_{L} h_{a b}=-k^{2} h_{a b} .
$$

This problem arises in perturbations of the six-dimensional solution (black ring) $\times \mathbb{R}$. If there is a zero frequency mode solution to $\Delta_{L} h_{a b}=0$, then there is also a zero frequency mode solution to (9) with $k=0$. One can find this mode by setting $\omega=0$ and solving (9) for $k$ while varying the parameters until $k=0$. Indeed, this method has been used successfully in the past $[14,15]$.

For our purposes, we need not find the zero frequency mode of the static ring. We merely observe that there are solutions of (9) on the static ring with $\omega=0$ and $k \neq 0$ that are connected to the zero frequency mode of the static ring and are hence also connected to our desired GregoryLaflamme mode of the balanced ring.

Given this, we first solve (9) with $\omega=0$ on the static ring for $k$. This problem is much simpler than solving (7) directly. This is a linear eigenvalue problem in $k^{2}$. The static background and $\omega=0$ introduce extra symmetry and reduce the number of functions from ten to seven. A suitable definition of the perturbation functions also yields matrices for the eigenvalue problem that are purely real. Furthermore, we know (as we know for black strings) that the real, positive $k^{2}$ modes are connected to the zero frequency mode, significantly reducing our search space for physical modes. Attempting this problem for a particular static ring by $Q Z$ factorization with several grid resolutions yielded a single real, positive value of $k^{2}$.

Having obtained a single solution connected to the solutions we are after, we can proceed by repeated application of the Newton-Raphson method on several grid resolutions. Since the Gregory-Laflamme modes of the static ring are pure imaginary, we can increase $\Gamma=-i \omega$ until we find that $k=0$. This puts us on a solution of (7). From here, we can solve for $\omega$ while varying the ring parameters. We increase the rotation until the ring is balanced, and we have thus arrived on a desired solution. We henceforth take the black ring to be balanced and present our results as we vary the parameter $\nu$.

As a check, we have evaluated the Geroch-Held-Penrose scalars constructed in Ref. [22] and found nonzero values, confirming that this mode cannot be pure gauge. More details of our calculation and a number of numerical checks can be found in Supplemental Material [23].

Results.-Figures 1 and 2 present the imaginary and real parts, respectively, of the dimensionless quantity $\varpi \equiv$ $\omega /(2 \pi T)$ as a function of $\nu$. (Recall that thin rings have $0<\nu \leq 1 / 2$ and fat rings have $1 / 2 \leq \nu<1$.) Dots and squares represent different resolutions, where $(N+N) \times N$ refers to $N^{2}$ points per patch. The agreement between resolutions is reassuring. Data points below $\nu \sim 0.144$ and above $\nu \sim 0.52$ were discarded due to lack of numerical precision. (We discard values where the two resolutions differ by more than $0.1 \%$.) The vertical dashed line separates fat and thin rings, with the colored region corresponding to fat rings.

Our main result can be seen in Fig. 1, where positive $\operatorname{Im}(\varpi)$ indicates an instability. An instability seems to exist for all values of $\nu \lesssim 0.55$ (the upper bound is an extrapolation of data with $\nu>0.3$ using a second-order polynomial in $\nu$ ). In particular, this instability extends into a region of the fat rings with $0.5 \leq \nu \lesssim 0.55$. Since all fat rings are already unstable to axisymmetric perturbations [18-20], this would imply that the Emparan-Reall ring is unstable for all ranges of parameters. We stress that, though we do not have points below $\nu \lesssim 0.144$, the Gregory-Laflamme argument should be valid for small $\nu$, so we expect the instability to persist down to $\nu=0$.

We note that the local Penrose inequalities suggest that the axisymmetric $(m=0)$ instability should be marginal at $\nu=1 / 2$, so fat rings near $\nu=1 / 2$ should have a small growth rate. Our results therefore suggest that the $m=2$ mode is dominant over the $m=0$ modes for fat rings near $\nu=1 / 2$. For larger values of $\nu$, the $m=2$ modes are no longer unstable, so the $m=0$ modes should dominate. Finding the transition point would requires data on the $m=0$ sector of perturbations, which we leave for future work.

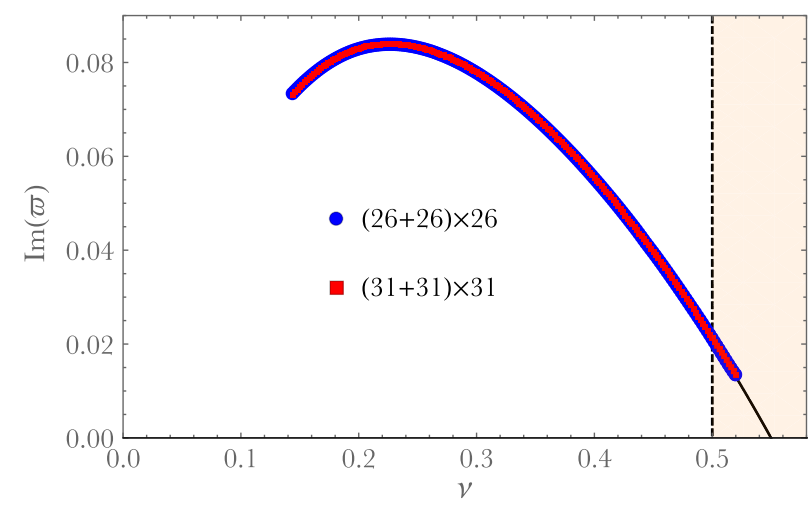

FIG. 1 (color online). Imaginary part of the frequency $\varpi \equiv$ $\omega /(2 \pi T)$ for nonaxisymmetric perturbations with $m=2$ as a function of $\nu$ at two resolutions. The colored region corresponds to fat rings which have previously been shown to be unstable. The solid line is a polynomial extrapolation. 


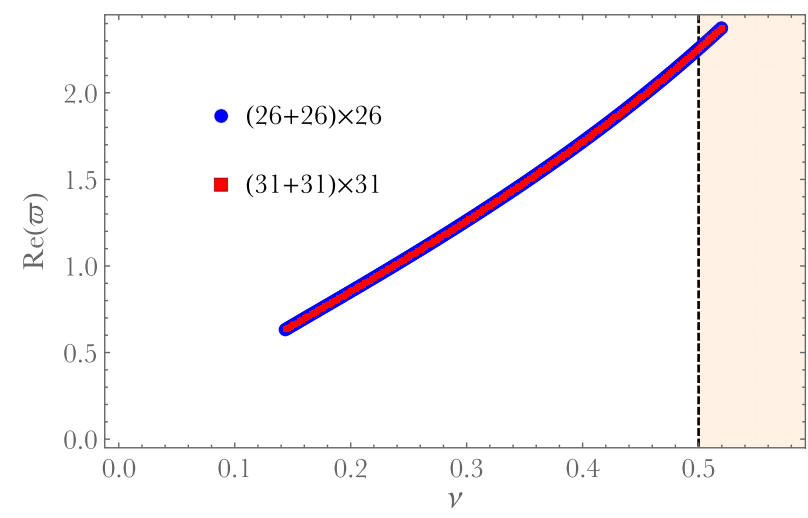

FIG. 2 (color online). Real part of the frequency $\varpi \equiv \omega /(2 \pi T)$ as a function of $\nu$. The same color scheme as Fig. 1 .

A curious fact about Fig. 2 involves the so-called superradiant bound. Based on the results of Refs. [24,25], the onset of the instability must satisfy $0 \leq \operatorname{Re}(\omega) \leq m \Omega$, as we have verified. Away from the onset at $\nu \lesssim 0.305$, we find instead that $\operatorname{Re}(\omega)>m \Omega$. Though this is not in conflict with Refs. [24,25], we do not have many examples where $\operatorname{Im}(\omega)>0$ and $\operatorname{Re}(\omega)>m \Omega$. The physical significance of this property is unclear at the moment.

To visualize the horizon evolution under this instability, we have constructed an isometric embedding of our perturbations (see Supplemental Material [23] for more details). The results are depicted in Fig. 3 and are similar to those obtained in Ref. [26] for the Gregory-Laflamme instability. This corroborates our claim that these nonaxisymmetric perturbations are similar to that of the black string. The general shape of the curve in Fig. 1 is also characteristic of Gregory-Laflamme modes [13,21].

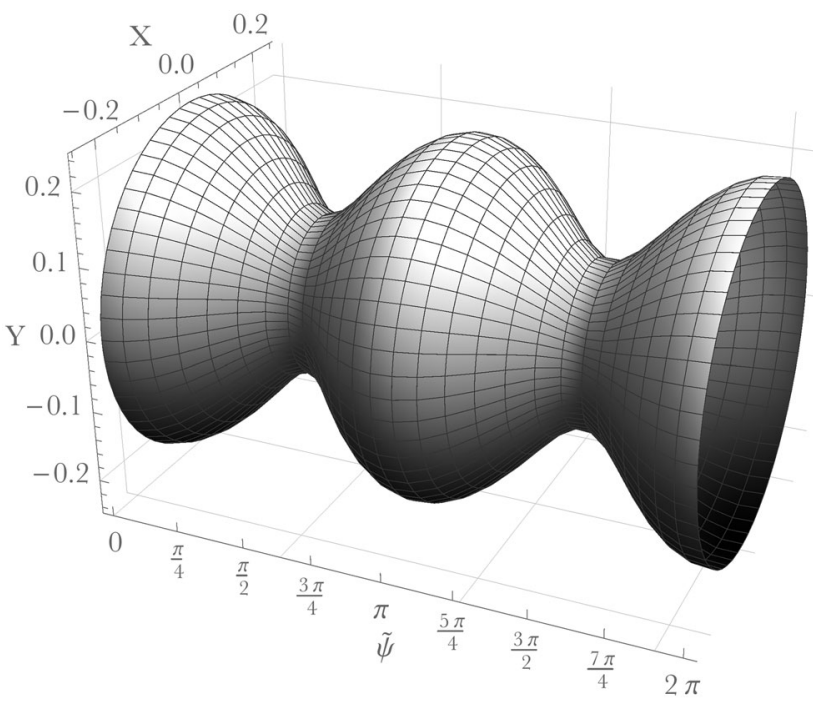

FIG. 3. Isometric embedding of constant $\psi$ slices of the $S^{2}$ spatial sections of the perturbed black ring horizon (see Supplemental Material [23] for more details). This plot corresponds to $\nu=0.2$.
Outlook.-We have given substantial numerical evidence that the entire Emparan-Reall black ring is unstable. Fat rings are unstable to axisymmetric perturbations, and thin rings are unstable to nonaxisymmetric perturbations resembling the Gregory-Laflamme instability of the black string. There is a competition between these instabilities for fat rings near $\nu=1 / 2$.

We focused our computation entirely on the $m=2$ modes. It would be interesting to see how the other modes behave. A study of the $m=0$ modes would elucidate its competition with the $m=2$ modes. There could also be instabilities corresponding to $m=1$, though these were absent in Myers-Perry black holes [10]. Perturbations with $m>2$ correspond to a shorter wavelength and may have an onset for smaller $\nu$ than that of $m=2$. One can also consider perturbations that break the $\partial_{\phi}$ symmetry.

While we have studied only stability of the EmparanReall black ring, there are many other solutions. In five dimensions, the black ring can also rotate in the $\partial_{\phi}$ direction as in the double-spinning ring [27] and the helical rings [28]. A study of rotating black strings [15] suggests that the instability in doubly spinning rings would have a higher growth rate. There are also multihorizon solutions (e.g., black Saturns [29], dirings [30,31], and bicycling rings [32]). These solutions contain black rings as horizon components and might share many of the same stability properties. In six and higher dimensions, there are also black rings [33,34] and their associated multihorizon solutions, but there are additionally ringoids $[28,35]$ and lumpy black holes [34,36]. Little is known about the stability of these solutions, though many of them resemble an unstable Myers-Perry black hole, or a black ring, and hence might also share many stability properties. The addition of matter may add a stabilizing effect, particularly in supersymmetric setups [37-40].

The end point of these instabilities remains an important and open problem. Work is in progress [41]. Axisymmetric instabilities are expected to lead towards Myers-Perry black holes. For nonaxisymmetric perturbations of very thin rings, the Gregory-Laflamme instability in the (unboosted) black string suggests that black rings would develop a naked singularity and violate cosmic censorship [42]. The similarity of our results to the Gregory-Laflamme instability seems to support this idea. It is important to note, however, that this instability in the black ring emits gravitational radiation. Even for very thin rings, the solutions resemble boosted black strings, and it is unclear what role this would play in the overall time evolution.

We thank Joan Camps, Gary Horowitz, and Donald Marolf for helpful discussions, Óscar Dias for comments on a draft of this Letter, and Harvey Reall for comments and for being such a good sport. B.W. is supported by European Research Council Grant No. ERC-2011-StG 279363-HiDGR. 
*J.E.Santos@damtp.cam.ac.uk

†B.Way@damtp.cam.ac.uk

[1] R. P. Kerr, Gravitational Field of a Spinning Mass as an Example of Algebraically Special Metrics, Phys. Rev. Lett. 11, 237 (1963).

[2] D. Robinson, in The Kerr Spacetime: Rotating Black Holes in General Relativity, edited by D. L. Wiltshire, M. Visser, and S. M. Scott (Cambridge University Press, Cambridge, England, 2009).

[3] P. T. Chrusciel, J. L. Costa, and M. Heusler, Stationary black holes: Uniqueness and beyond, Living Rev. Relativity 15, 7 (2012).

[4] B. F. Whiting, Mode stability of the Kerr black hole, J. Math. Phys. (N.Y.) 30, 1301 (1989).

[5] M. Dafermos and I. Rodnianski, Decay for solutions of the wave equation on Kerr exterior spacetimes I-II: The cases $|a| \ll M$ or axisymmetry, arXiv:1010.5132.

[6] M. Dafermos, I. Rodnianski, and Y. Shlapentokh-Rothman, Decay for solutions of the wave equation on Kerr exterior spacetimes III: The full subextremal case $|a|<M$, arXiv:1402.7034.

[7] M. Dafermos, I. Rodnianski, and Y. Shlapentokh-Rothman, A scattering theory for the wave equation on Kerr black hole exteriors, arXiv:1412.8379.

[8] R. C. Myers and M. Perry, Black holes in higher dimensional space-times, Ann. Phys. (N.Y.) 172, 304 (1986).

[9] M. Shibata and H. Yoshino, Bar-mode instability of rapidly spinning black hole in higher dimensions: Numerical simulation in general relativity, Phys. Rev. D 81, 104035 (2010).

[10] O. J. Dias, G. S. Hartnett, and J. E. Santos, Quasinormal modes of asymptotically flat rotating black holes, Classical Quantum Gravity 31, 245011 (2014).

[11] R. Emparan and H.S. Reall, A Rotating Black Ring Solution in Five Dimensions, Phys. Rev. Lett. 88, 101101 (2002).

[12] R. Emparan and H. S. Reall, Black rings, Classical Quantum Gravity 23, R169 (2006).

[13] R. Gregory and R. Laflamme, Black Strings and p-Branes are Unstable, Phys. Rev. Lett. 70, 2837 (1993).

[14] O. J. Dias, P. Figueras, R. Monteiro, J. E. Santos, and R. Emparan, Instability and new phases of higher-dimensional rotating black holes, Phys. Rev. D 80, 111701 (2009).

[15] O. J. Dias, P. Figueras, R. Monteiro, H. S. Reall, and J. E. Santos, An instability of higher-dimensional rotating black holes, J. High Energy Phys. 05 (2010) 076.

[16] M. Shibata and H. Yoshino, Nonaxisymmetric instability of rapidly rotating black hole in five dimensions, Phys. Rev. D 81, 021501 (2010).

[17] G.S. Hartnett and J. E. Santos, Non-axisymmetric instability of rotating black holes in higher dimensions, Phys. Rev. D 88, 041505 (2013).

[18] G. Arcioni and E. Lozano-Tellechea, Stability and critical phenomena of black holes and black rings, Phys. Rev. D 72, 104021 (2005).

[19] H. Elvang, R. Emparan, and A. Virmani, Dynamics and stability of black rings, J. High Energy Phys. 12 (2006) 074.
[20] P. Figueras, K. Murata, and H. S. Reall, Black hole instabilities and local Penrose inequalities, Classical Quantum Gravity 28, 225030 (2011).

[21] J. Hovdebo and R. C. Myers, Black rings, boosted strings and Gregory-Laflamme, Phys. Rev. D 73, 084013 (2006).

[22] M. Godazgar and H. S. Reall, Peeling of the Weyl tensor and gravitational radiation in higher dimensions, Phys. Rev. D 85, 084021 (2012).

[23] See Supplemental Material at http://link.aps.org/ supplemental/10.1103/PhysRevLett.114.221101 for further details of our calculation and some numerical checks.

[24] S. Teukolsky and W. Press, Perturbations of a rotating black hole. III-Interaction of the hole with gravitational and electromagnetic radiation, Astrophys. J. 193, 443 (1974).

[25] R. M. Wald, General Relativity (Chicago University, Chicago, 1984), Chap. 12.

[26] R. Gregory and R. Laflamme, The instability of charged black strings and p-branes, Nucl. Phys. B428, 399 (1994).

[27] A. Pomeransky and R. Sen'kov, Black ring with two angular momenta, arXiv:hep-th/0612005.

[28] R. Emparan, T. Harmark, V. Niarchos, and N. A. Obers, New horizons for black holes and branes, J. High Energy Phys. 04 (2010) 046.

[29] H. Elvang and P. Figueras, Black Saturn, J. High Energy Phys. 05 (2007) 050.

[30] H. Iguchi and T. Mishima, Black di-ring and infinite nonuniqueness, Phys. Rev. D 75, 064018 (2007).

[31] J. Evslin and C. Krishnan, The black di-ring: An inverse scattering construction, Classical Quantum Gravity 26, 125018 (2009).

[32] H. Elvang and M. J. Rodriguez, Bicycling black rings, J. High Energy Phys. 04 (2008) 045.

[33] B. Kleihaus, J. Kunz, and E. Radu, Black rings in six dimensions, Phys. Lett. B 718, 1073 (2013).

[34] O. J. Dias, J. E. Santos, and B. Way, Rings, ripples, and rotation: Connecting black holes to black rings, J. High Energy Phys. 07 (2014) 045.

[35] B. Kleihaus, J. Kunz, and E. Radu, Black ringoids: Spinning balanced black objects in $d \geq 5$ dimensions-The codimension-two case, J. High Energy Phys. 01 (2015) 117.

[36] R. Emparan, P. Figueras, and M. Martinez, Bumpy black holes, J. High Energy Phys. 12 (2014) 072.

[37] H. Elvang, R. Emparan, D. Mateos, and H. S. Reall, A Supersymmetric Black Ring, Phys. Rev. Lett. 93, 211302 (2004).

[38] I. Bena and N. P. Warner, One ring to rule them all ... and in the darkness bind them?, Adv. Theor. Math. Phys. 9, 667 (2005).

[39] H. Elvang, R. Emparan, D. Mateos, and H. S. Reall, Supersymmetric black rings and three-charge supertubes, Phys. Rev. D 71, 024033 (2005).

[40] J. P. Gauntlett and J. B. Gutowski, General concentric black rings, Phys. Rev. D 71, 045002 (2005).

[41] K. Clough, P. Figueras, H. Finkel, M. Kunesch, E. Lim, and S. Tunyasuvunakool (to be published).

[42] L. Lehner and F. Pretorius, Black Strings, Low Viscosity Fluids, and Violation of Cosmic Censorship, Phys. Rev. Lett. 105, 101102 (2010). 